\title{
Archivos de Cardiología de México
}

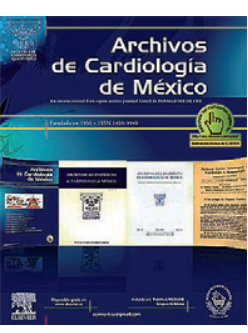

\section{ÍNDICE GENERAL DE AUTORES}

Agüero Sánchez Alejandro

Ahmad Mansoor

Aladro Miranda Iguer F.

Alcántara Razo Mónica

Alcocer Alejandro

Alcocer Gamba Marco Antonio

Alexánderson Rosas Erick

Alfonso Fernando

Álvarez Sangabriel Amada

Ambrosio Giuseppe

Amezcua Guerra Luis M.

Amigo Mary Carmen

Amores Arellano Nelson

Andreu José Manuel

Antoniades Loizos

Apetrei Eduard

Arai Kaduo

Arias Mendoza Alexandra

Arguedas Jiménez Hugo

Artigou Jean-Yves

Aschermann Michael

Bacilio Pérez Ulises

Barajas Gamboa Juan S.

Baranchuk Adrian

Basmadjian Arsène J.

Becerra Luna Brayans

Beltrán Gámez Miguel

Berenfeld Omer

Berman Sofia G.

Berti Marco

Böhm Michael

Bolognese Leonardo

Buendía Hernández Alfonso

Bugiardini Raffaele

Burelle Denis

Bustamante John

Caballero Caballero Rocío

Calvo Conrado

Calvo Naiara

Canepa Juan Pablo

Cantamessa Paola

Cárdenas Loaeza Manuel
Carrillo Calvillo Jorge $\quad 218$

Carrillo Córdova Jorge R. 204

Carrillo Sánchez Silvia 208

Castillo Genaro 135

Castrejón Sergio 139

Chain Sergio

Cruz López Diez Juan 324

Cohen Ariel 170

Chávez Domínguez Rafael César 262

Damas de los Santos Félix 7, 105

Dávila Rodrigo 197

De la Garza Paola 48

De Micheli Alfredo 153, 252

Delgadillo Hilda 208

Del Pino Enrique J. 1

Del Valle Zamora Karina $\quad 48$

Deras Mejía Luz María 282

Díaz Pérez Julio A. $\quad 214$

Doiny David 139

Dore Annie $\quad 37$

Ducharme Anique $\quad 37$

Echavarría Pinto Mauro 195

Ector Hugo $\quad 170$

Edes Istvan $\quad 170$

Eid Lidt Guering 7, 105, 303

Elias Joseph 170

Elliott Perry M. 59

Enríquez Gómez Edmundo $\quad 120$

Escobedo Uribe Carlos David 218

Espinola Zavaleta Nilda 290

Esquinca Vera Juan Carlos $\quad 105$

Estañol Bruno 91

Estrada Alejandro $\quad 139$

Franco Martha $\quad 312$

Feldman Gabriela $\quad 1$

Femenia Francisco $\quad 324$

Figueroa Solano Javier $\quad 153$

Filgueiras Rama David $\quad 139$

Fragoso José Manuel 208 
Galeano Javier

Gálvez Pérez Faustino

Gamboa Ricardo

García Badillo Edgar

García Bolao Ignacio

García de Yébenes Manuel

García Graullera Juan Manuel

García Hernández Enrique

García Navarro Miguel

Garceau Patrick

Gaspar Hernández Jorge

Gavira Juan José

Ghizzoni Giuseppe

Giachello Federico

Giugliano Robert P.

Gómez Monterrosas Omar

González Chinea Ramón

González Gómez Carlos A.

González Pacheco Héctor

González Toledo Rafael

González Vidal

Grau Abalo Ricardo

Grip Laura T.

Grupo de investigadores participantes en

México para el estudio I-Search

Gualeni Anna

Guarda Eduardo

Haouala Habib

Heras Magda

Herrera Ramón N.

Höglund Christer

Huber Kurt

Hulin Ivan

Ibargollín Hernández Rosendo S.

Ibarra Castillo Rita

Ibrahim Reda

Ilarraza Lomelí Hermes

Infante Óscar

Ivanusa Mario

Iturralde Torres Pedro

Izaguirre Ávila Raúl

Jalife José

Juárez Herrera Úrsulo

Kimura Hayama Eric

Krittayaphong Rungroj

Kulakowski Piotr

Kuo Chi-Tai

Kuri Magdalena

Lara Pérez Norge R.

Lau Chu-Pak

Leiva Pons José Luis

León Camargo Yoana
170

320

34

14

235

235

125

303

248

37

105

235

22

34

308

208

297

214

7

312

135

73,112

308

93

22

170

170

170

1

170

170

170

297

131

37

153, 160

82,197

170

252

252

139

7, 14

105, 195

170

170

170

273

297

170

218

214
Lerma Claudia

82

Lomelí Catalina

93

López Jiménez Francisco

López Quijano Juan Manuel

Lorenzo Antonio

218

Lucas Luciardi Héctor

Lugo Gavidia Leslie M.

Lüscher Thomas $F$.

Lyusov Victor A.

Macías Garrido Enrico

Marcotte François

Marín Francisco

Marinskis Germanas

Márquez Murillo Manlio F.

Martínez Chávez Jorge Alberto

Martínez García Paola

Martínez Lavín Manuel

Martínez Memije Raúl

Martínez Reding Jesús

Martínez Ríos Marco Antonio

Martínez Rodríguez Nancy

Martínez Sánchez Carlos R.

Masic Izet

Meaney Eduardo

Meave González Aloha

Mercier Lise-Andrée

Merino José L.

Merino Pasaye Laura Elizabeth

Miranda Chávez Irma

Mohiddin Saidi A.

Monsiváis Urenda Adriana Elizabeth

Montalvo Ramos Aquiles

Morales Quispe Jorge Alberto

Moreno Galdós Leire

Moreno Martínez Francisco L.

Mrochek Alexander

Muntaner Juan A.

Muñoz Castellanos Luis

Nodarse Valdivia José R.

Ochoa Juan Manuel

Oganov Rafael G.

O'Meara Eileen

Oneglia Carlo

Ortega Marta

Pacheco Bouthillier Alex

Pavón Martínez Natalia

Pesce Ricardo

Pelletier Guy B.

Peña Duque Marco Antonio

Pérez Baztarrica Gabriel

Pérez Fernández Guillermo A.

Pérez González Alberto

Pérez Méndez Oscar
79, 170, 243

303

82

$1,7,14,120$

120, 125

37

139

290

153

218

303

290

235

297

170

$73,112,260$

105

208 
Pinho Moreira Luiz Felipe

170

170

Pinto Fausto J.

Porcile Rafael

Pulido Zamudio Tomás

Raev Dimitar

Ramírez Fuentes Silvestre

Ramírez Gutiérrez Álvaro Eduardo

Ramírez Neria Paulina

Reyes Fuentes Luis Felipe

Ricalde Alcocer Alejandro

Ríos Méndez Raúl E.

Rius María Dolores

Rivera Capello Juan M.

Rødevand Olaf

Rodríguez Armando

Rodríguez Escudero Juan Pablo

Rodríguez Ortega Fernando

Rodríguez Piña Horacio

Rodríguez Rodríguez Engels

Rogava Mamanti

Rojas Velasco Gustavo

Roldán Gómez Francisco Javier

Roldán Vanessa

Rosado Carolina

Rosas Peralta Martín

Rosenhek Raphael

Ruipérez Juan Antonio

Saavedra Daniel

Salgado Paola

Salvaggio Flavio

Sánchez Díaz Carlos Jerjes

Sánchez Ramírez Carlos Jerjes

105, 125

48

48

125

34

153

135

170

48

66

290

282

195

170

120

320,326

248

48

93

181

248
Sandoval Julio 48

Sandoval Gómez Cecilia

214

Sansoy Vedat

Sarmiento Ricardo A.

Saucedo Nestor

170

34

93

Shimokawa Hiroaki

170

Shumakov Valentin A. $\quad 170$

Sobrino Ayax 37

Solano Gutiérrez Alejandro 290

Soldán Patiño Claudia

Patricia Paz

125

Solernó Raúl

Stefanadis Christodoulos

34

170

Tajer Carlos Daniel

170

Téllez Jorge $\quad 48$

Timmis Adam $\quad 170$

Torres Quiroz Ivonne Aimeé $\quad 303$

Trejo Gutiérrez Jorge F. 66

Valbuena Javier $\quad 265$

Valdés Mariano 248

Valero Elina 230

Van der Wall Ernst E. $\quad 170$

Vardas Panos 170

Vargas Alarcón Gilberto 208

Vargas Barrón Jesús $\quad 120,320$

Veja Fleites Luis F. 297

324 Velázquez Rodríguez Enrique 282

197 Velázquez Verduzco Arturo 303

31 Videbæk Jørgen 170

14 Videla Lynch Ángeles 34

14 Villecco Sebastián 31 